\title{
Rmetrics - timeDate Package
}

by Yohan Chalabi, Martin Mächler, and Diethelm Würtz

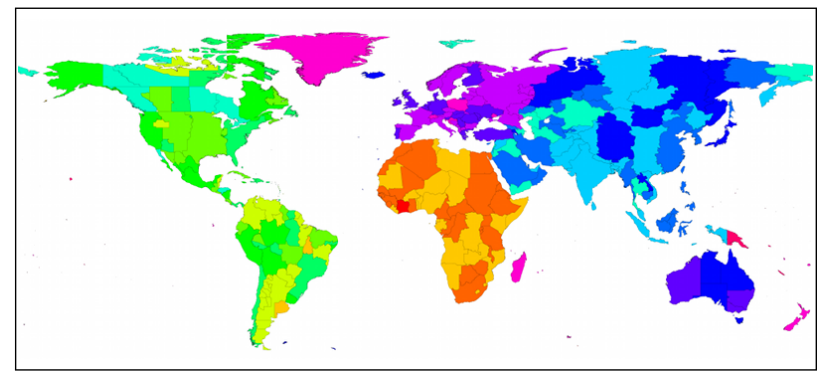

Figure 1: World map with major time zones. ${ }^{1}$

\begin{abstract}
The management of time and holidays can prove crucial in applications that rely on historical data. A typical example is the aggregation of a data set recorded in different time zones and under different daylight saving time rules. Besides the time zone conversion function, which is well supported by default classes in $\mathrm{R}$, one might need functions to handle special days or holidays. In this respect, the package timeDate enhances default date-time classes in $\mathrm{R}$ and brings new functionalities to time zone management and the creation of holiday calendars.
\end{abstract}

Chronological data sets recorded in different time zones play an important role in industrial applications. For example, in financial applications, it is common to aggregate time series that have been recorded in financial centers with different daylight saving time (DST) rules and time zones.

$\mathrm{R}$ includes different classes to represent dates and time. The class that holds particular interest for us is the "POSIXct" one. It internally records timestamps as the number of seconds from "197001-01 UTC", where UTC stands for universal time coordinated. Moreover, it supports the DST and time zone functions by using the rules provided by the operating system (OS). However, at the time timeDate (Würtz et al. (2011)) - formerly known as fCalendar-was first released, the implementation of the DST function was not consistent across OSs. Back then, the main purpose of the package was to have DST rules directly available to bring consistency over OSs. Today, DST support by OSs is not a problematic question as it used to be. As we will show later, the "timeDate" class is based on the "POSIXct" one. Both classes hence share common functionalities. However, the timeDate package has some additional functionalities, which we will emphasize in this note. For related date-time classes in
R, see Ripley \& Hornik (2001) and Grothendieck \& Petzoldt (2004).

Another problem commonly faced in managing time and dates is the midnight standard. Indeed, the problem can be handled in different ways depending on the standard in use. For example, the standard C library does not allow for the "midnight" standard in the format "24:00:00" (Bateman (2000)). However, the timeDate package supports this format.

Moreover, timeDate includes functions for calendar manipulations, business days, weekends, and public and ecclesiastical holidays. One can handle day count conventions and rolling business conventions. Such periods can pertain to, for example, the last working day of the month. The below examples illustrate this point.

In the remaining part of this note, we first present the structure of the "timeDate" class. Then, we explain the creation of "timeDate" objects. The use of financial centers with DST rules is described next. Thereafter, we explain the management of holidays. Finally, operations on "timeDate" objects such as mathematical operations, rounding, subsetting, and coercions are discussed. Throughout this note, we provide many examples to illustrate the functionalities of the package.

\section{Structure of the "timeDate" class}

The "timeDate" S4 class is composed of a "POSIXct" object that is always in Greenwich Mean Time (GMT) and of a financial center that keeps track of DST. We use the term financial center to denote geographical locations. This terminology stems from the main application of the Rmetrics packages. However, this denomination should not stop programmers from using the package in other fields. By default, the local financial center is set to GMT. The default setting can be changed via setRmetrics0ptions (myFinCenter $=$ ....). The formal $\mathrm{s} 4$ class is defined as

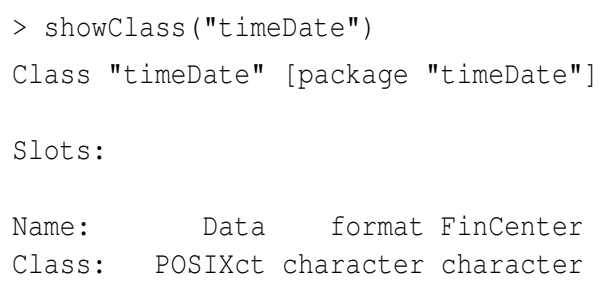

where the slot Data contains the timestamps in the POSIXct class, format is the format typically applied to Data, and FinCenter is the financial center.

Note: we use the abbreviation GMT equivalently to UTC, the universal time coordinated.

\footnotetext{
${ }^{1}$ The original data set of the world map with time zones is available at http://efele.net/maps/tz/world/. Full and reduced rda versions were kindly contributed by Roger Bivand.
} 


\section{"timeDate" object creation}

There are different ways to generate a "timeDate" object. It can be generated using either timeDate (), timesequence (), or timeCalendar ().

The function timeDate () creates a "timeDate" object from scratch. It requires a character vector of timestamps and optional arguments to specify the format of this vector and the financial center. The financial center can be specified, as mentioned, via setRmetricsoptions () or (more cleanly) with the argument FinCenter. By default, it is set to GMT².

In the following, we illustrate the creation of "timeDate" objects as well as the method used to convert timestamps from different time zones.

We first create character vectors of two timestamps with the default financial center (GMT):

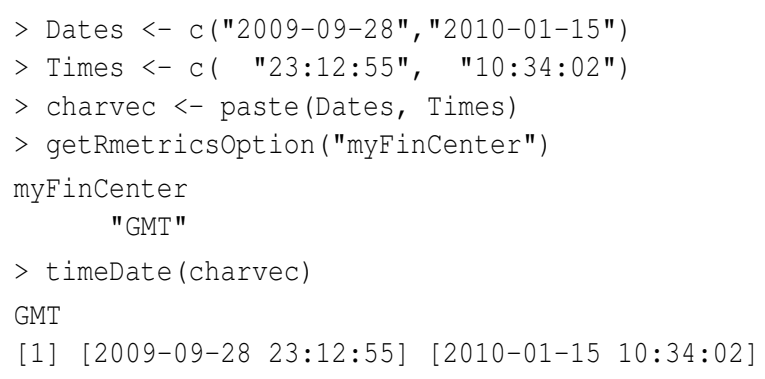

As a second step, we set the local financial center to Zurich and create a "timeDate" object.

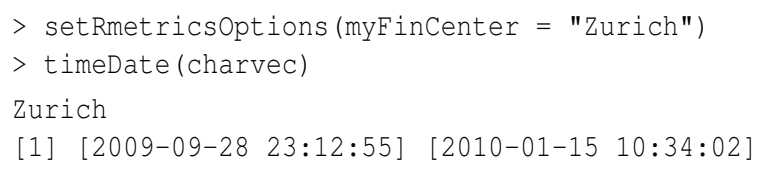

The third example shows how the timestamps can be conveniently converted into different time zones; charvec is recorded in Tokyo and subsequently converted to our local center, i.e., Zurich (see above):

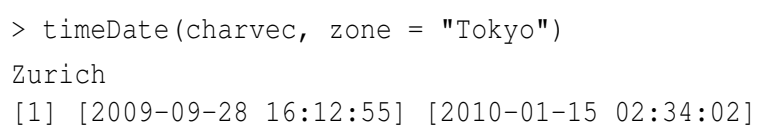

It is also possible to use the function fincenter () to view or modify the local center:

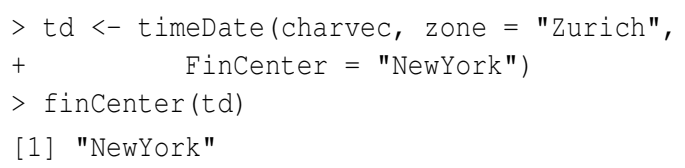

\footnotetext{
${ }^{2}$ GMT can be considered as a "virtual" financial center.
}

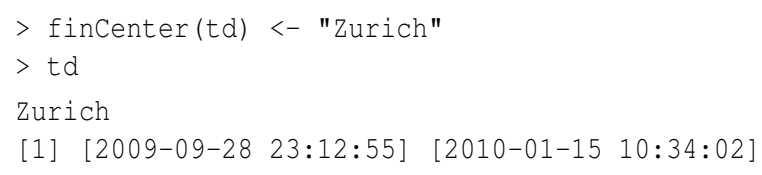

If the format of charvec is not specified, timeDate uses an automated date-time format identifier called whichFormat () that supports common date-time formats.

$>$ whichFormat (charvec)

[1] "ำ $-\frac{\circ}{0}-\circ \mathrm{d} \% \mathrm{H}: \% \mathrm{M}: \% \mathrm{~S} "$

The function timesequence() creates a "timeDate" object representing an equidistant sequence of points in time. You can specify the range of dates with the arguments from and to. If from is missing, length.out defines the length of the sequence. In the case of a monthly sequence, you can define specific rules. For example, you can generate the sequence with the last days of the month or with the last or n-th Friday of every month. This can be of particular interest in financial applications.

Let us first reset the financial center to an international environment:

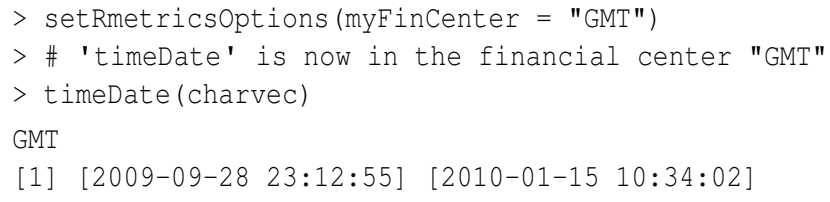

A sequence of days or months can be created as follows:

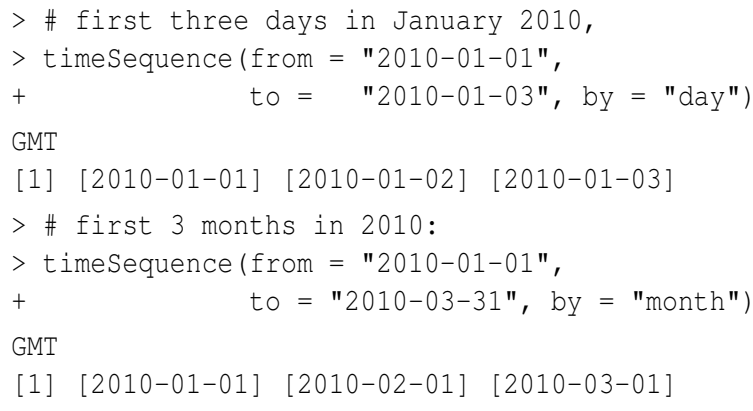

The function timeCalendar() creates "timeDate" objects from calendar atoms. You can specify values or vectors of equal length denoting year, month, day, hour, minute, and seconds as integers. For example, the monthly calendar of the current year or a specific calendar in a given time zone can be created as follows:

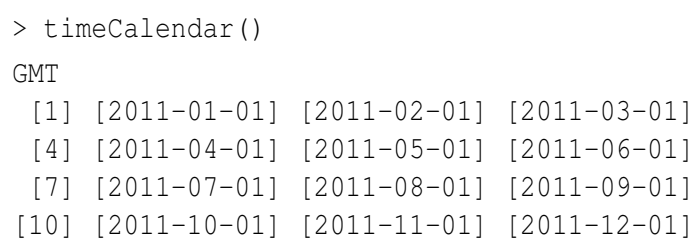


The following represents the first four days of January recorded in Tokyo at local time "16:00" and converted to the financial center Zurich:

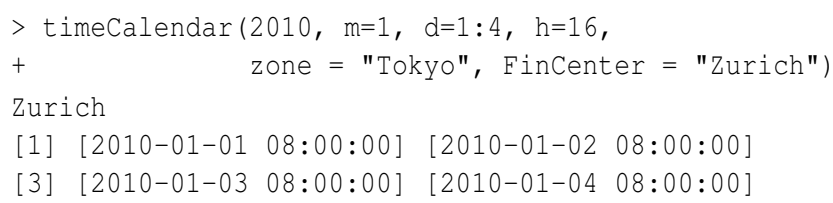

\section{Midnight standard}

The "timeDate" printing format is designed in accordance with the ISO-8601 (1988) standard. It uses the 24-hour clock format. Dates are expressed in the "\%Y-\%m-\%d" format while time-dates are stated in the "\%Y-\%m-\%d \%H:\%M:\%S" format. A special case in the 24-hour clock system is the representation of midnight. It can be equivalently represented by "00:00" and "24:00". The former is usually used to denote the beginning of the day whereas the latter denotes the end. timeDate supports the midnight standard as described by the ISO-8601 (1988) standard as illustrated here:

$>$ timeDate (ch <- "2010-01-31 24:00:00")

GMT

\section{[1] [2010-02-01]}

Note, following the revision of this paper, the $R$ source has been amended in May 2011 to also allow "24:00" time specifications, such that a midnight standard will be part of standard R.

\section{Financial centers - via daylight sav- ing time rules}

As mentioned earlier, the global financial center can be set with the function setRmetricsoptions () and accessed with the function getRmetricsOption(). Its default value is set to "GMT":

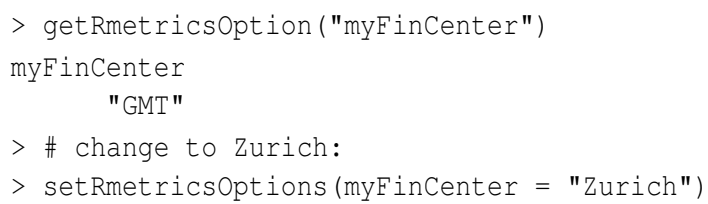

From now on, all dates and times are handled in accordance with the Central European time zone and the DST rule for Zurich. Note that setting the financial center to a continent/city that lies outside the time zone used by your OS does not change any of the time settings or environment variables of your computer.

There are almost 400 financial centers supported thanks to the Olson database. They can be accessed by the function listFincenter(), and partial lists can be extracted through the use of regular expressions.

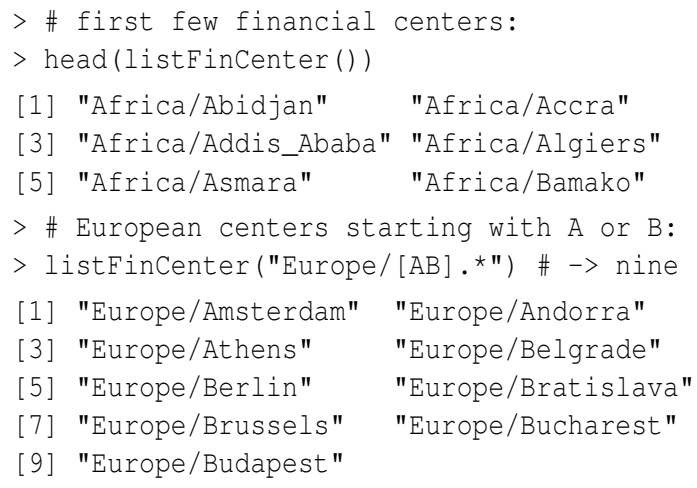

Each financial center has an associated function that returns its DST rules in the form of a "data.frame". These functions share the name of their financial center, e.g., Zurich ().

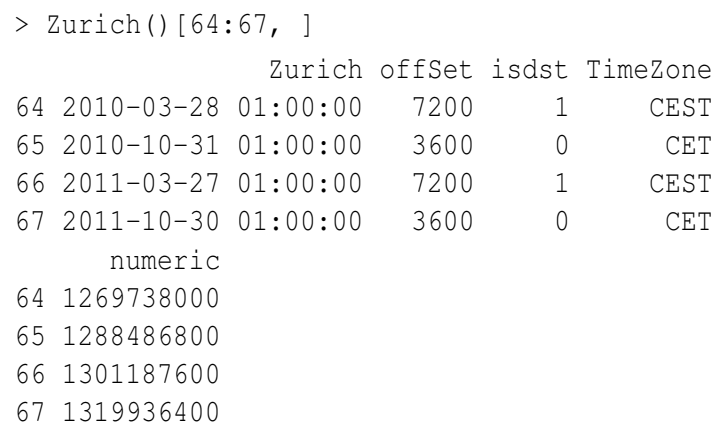

The returned "data.frame" shows when the clock was changed in Zurich, the offset in seconds with respect to GMT, a flag that tells us if DST is in effect or not, the time zone abbreviation, and the number of seconds since "1970-01-01" in GMT. The reader interested in the history of DST is referred to Bartky \& Harrison (1979).

Note new centers can be easily added as long as their associated functions return a "data.frame" with the same structure as described above.

\section{Holidays and calendars}

Holidays are usually grouped by their origins. The first category, as the etymology suggests, is based on religious origin. For example, the ecclesiastical calendars of Christian churches are based on cycles of movable and immovable feasts. Christmas, December 25, is the principal immovable feast, whereas Easter is the principal movable feast. Most of the other dates are movable feasts that are determined with respect to Easter, Montes (1996). A second category of holidays is secular holidays, which denotes days that are celebrated internationally and in different cultures, such as Labor Day. Another category of holidays includes days that are relative to natural events. For example, the dates can be related to astronomical events such as cycles of the moon or the equinox. Moreover, there are also country-specific national holidays. 
The calculation of holidays might prove tedious in some circumstances. Indeed, the estimation of the Easter date is a complex procedure with different algorithms involved in its computation. The algorithm implemented in the package is the one of Oudin (1940) as quoted in Seidelmann (1992). This approach is valid for any Gregorian calendar year. Further details about holiday calculation can be found in Tøndering (2008).

The dates of Easter for the next five years can be calculated with

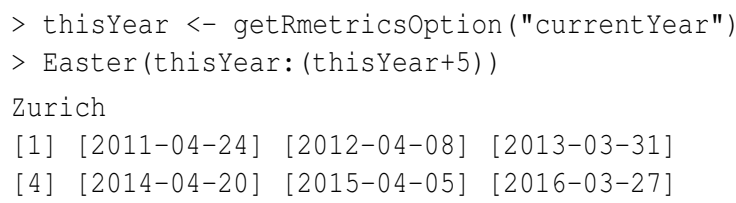

The timeDate package includes functions for bank holidays in Canada, France, Germany, Great Britain, Italy, Japan ${ }^{3}$, Switzerland, and the US. These holidays can be grouped in calendars. At the moment, the package provides functions for the New York stock exchange, holidayNYSE (); for the North American Reliability Council, holidayNERC () ${ }^{4}$; for the Toronto stock exchange holidayTSX(), and for Zurich, holidayZURICH(). Other calendars can be easily implemented given that the package already provides many holidays functions. A list of all holidays is provided in the appendix.

\section{Logical test}

It is possible to construct tests for weekdays, weekends, business days, and holidays with the functions isWeekday(), isWeekend(), isBizday() and isHoliday (), respectively.

Let us take a sequence of dates around Easter:

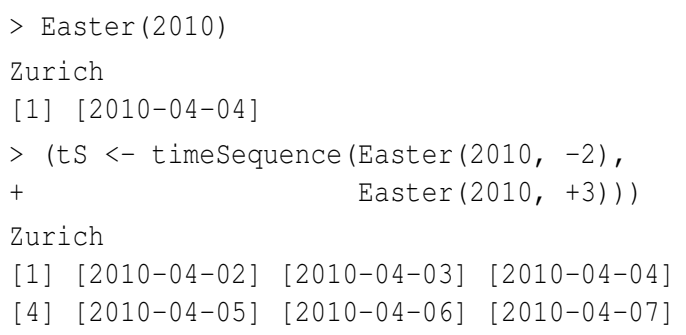

We can now extract the weekdays or business days according to a holiday calendar.

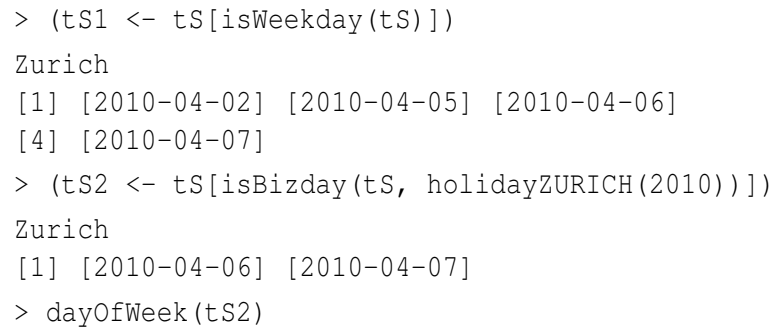

\footnotetext{
${ }^{3}$ The Japanese holidays were contributed by Parlamis Franklin.

${ }^{4}$ holidayNERC () was contributed by Joe W. Byers.
}

2010-04-06 2010-04-07

"Tue" "Wed"

Thank to the comments of one of the referees, we have added a new argument, wday, in the functions isWeekend(), isWeekday(), isBizday() and isHoliday () that can be used to specify which days should be considered as business days. This is important when using calendars in Islamic countries or in Israel. By default, wday specifies the weekdays as Monday to Friday.

\section{Special dates}

As mentioned earlier, holidays often refer to a specific date or event. It is therefore crucial to have functions to compute: the first day in a given month, the last day in a given month and year, a given day before or after a date, the n-th occurrences of a day in a specified year/month, or a given last day for a specified year/month. We have summarized these functions in a table in the appendix.

In the following, we demonstrate how to retrieve the last day in each quarter or the second Sunday of each month. Note that days are numbered from 0 to 6 where 0 corresponds to Sunday and 6 to Saturday.

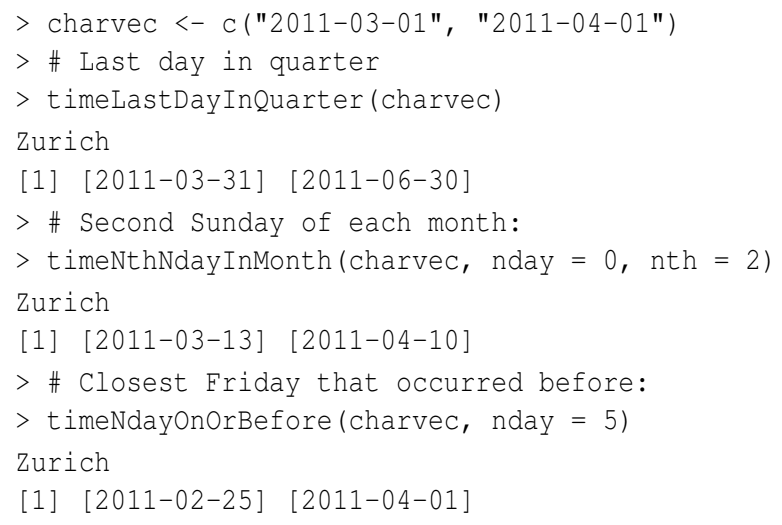

\section{Operations on "timeDate" objects}

Just like the other date-time classes in $\mathrm{R}$, the "timeDate" class supports common operations. It allows for mathematical operations such as addition, subtraction, and comparisons to be performed. Moreover, methods for the generic functions to concatenate, replicate, sort, re-sample, unify, revert, or lag are available as the well known calls $c(), \operatorname{rep}()$, sort (), sample(), unique (), rev(), and diff(), respectively. We spare the reader superfluous examples of functions that are common to other datetime classes. In the rest of the section, we emphasize methods that are not available for other classes or are not strictly identical. The reader is referred to the ebook "Chronological Objects with Rmetrics" (Würtz, Chalabi \& Ellis, 2010) for more examples. 


\section{Subsetting methods}

The timeDate package has different functions to subset a timeDate object. The usual function '[' extracts or replaces subsets of "timeDate" objects as expected. However, the package provides some additional functions. For example, the function window () extracts a sequence from a starting and ending point. The functions start () and end () extract the first and last timestamps, respectively.

\section{Coercion methods}

The package provides both $\mathrm{S} 3$ and $\mathrm{S} 4$ methods to coerce to and from "timeDate" objects. Below, we list the $\mathrm{S} 4$ coercion methods available. The equivalent $\mathrm{S3}$ methods as . * are also provided, although the mixing of S4 classes and S3 methods is discouraged. Note that information can be lost in the coercion process if the destination class does not support the same functionality.

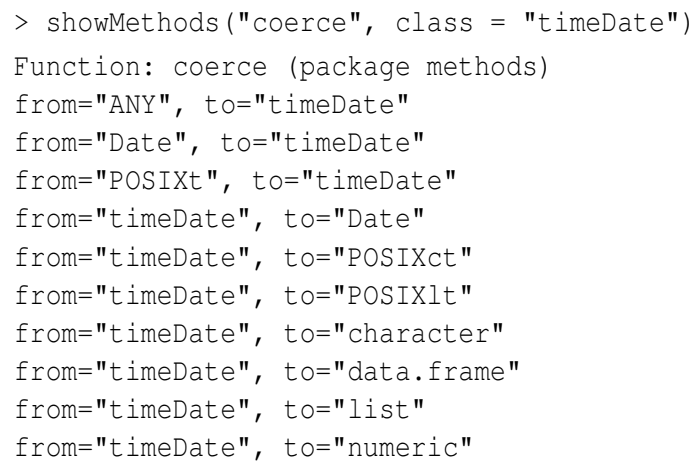

We would like to point out a particular difference between the as.numeric methods of "timeDate" and "POSIXct" classes. Indeed, the as .numeric-timeDate method returns the time in minutes, in contrast to as.numeric.POSIXct, which returns the results in seconds. However, the as.numeric-timeDate method has an additional argument, unit, to select other units. These include seconds, hours, days, and weeks.

\section{Concatenation method}

The concatenation c() method for "timeDate" objects takes care of the different financial centers of the object to be concatenated. In such cases, all timestamps are transformed to the financial center of the first "timeDate" object. This feature is now also supported by R's "POSIXct" class. However, it was not available in previous versions of the class.

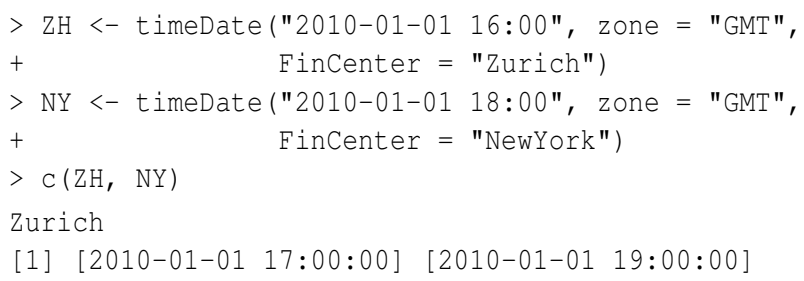

$>\mathrm{C}(\mathrm{NY}, \mathrm{ZH})$

NewYork

[1] [2010-01-01 13:00:00] [2010-01-01 11:00:00]

\section{Rounding and truncation methods}

The rounding and truncation methods have similar functionalities to those of their counterparts in other date-time classes. However, the default unit argument is not the same.

\section{Summary}

The timeDate package offers functions for calendar manipulations for business days, weekends, and public and ecclesiastical holidays that are of interest in financial applications as well as in other fields. Moreover, the Financial Center concept facilitates the mixing of data collected in different time zones and the manipulation of data recorded in the same time zone but with different DST rules.

\section{Acknowledgments}

We thank the anonymous referees and the editor for their valuable comments as well as all $R$ users and developers who have helped us to improve the timeDate package.

\section{Bibliography}

R.I. Bartky and E. Harrison. Standard and Daylight Saving Time. Scientific American, 240:46-53, 1979.

R. Bateman. Time Functionality in the Standard C Library, Novell AppNotes, September Issue, 73-85, 2000.

N. Dershowitz and E.M. Reingold. Calendrical Calculations. Software - Practice and Experience, 20:899928, 1990.

N. Dershowitz and E.M. Reingold. Calendrical Calculations. Cambridge University Press, 1997.

G. Grothendieck and T. Petzoldt. Date and Time Classes in R. R News, 4(1):29-32, 2004.

ISO-8601. Data Elements and Interchange Formats Information Interchange, Representation of Dates and Time. International Organization for Standardization, Reference Number ISO 8601, 1988.

M.J. Montes. Butcher's Algorithm for Calculating the Date of Easter in the Gregorian Calendar, 1996.

B.D. Ripley and K. Hornik. Date-Time Classes. $R$ News, 1(2):8-12, 2001. 
P.K. Seidelmann (Editor). Explanatory Supplement to the Astronomical Almanac. University Science Books, Herndon, 1992.

C. Tøndering. Frequently Asked Questions about Calendars, 2008. URL http: //www.tondering.dk/ claus/calendar.html.

D. Würtz and Y. Chalabi with contribution from M. Maechler, J.W. Byers, and others. timeDate: Rmetrics - Chronological and Calendarical Objects, 2011. URL http://cran.r-project.org/ web/packages/timeDate/.

D. Würtz, Y. Chalabi and A. Ellis Chronological Objects with Rmetrics, 2010. URL http://www . rmetrics.org/ebooks.

\section{Yohan Chalabi}

Finance Online $\mathrm{GmbH}$, Zurich $\mathcal{E}$

Institute of Theoretical Physics, ETH Zurich,

Switzerland

chalabiephys.ethz.ch

\section{Martin Mächler \\ Seminar für Statistik, ETH Zurich, \\ Switzerland \\ maechler@stat.math.ethz.ch}

\section{Diethelm Würtz}

Institute of Theoretical Physics, ETH Zurich, Switzerland

wuertz@phys.ethz.ch

\section{Appendix}

\section{Session Info}

$>$ toLatex(sessionInfo())

- $R$ version 2.13.0 (2011-04-13), x86_64-apple-darwin10.7.0

- Locale: C ...

- Base packages: base, datasets, grDevices, graphics, methods, stats, utils

- Other packages: timeDate 2130.93

\section{Tables}

\begin{tabular}{ll}
\hline 1. Special dates & \\
\hline timeFirstDayInMonth & First day in a given month \\
timeLastDayInMonth & Last day in a given month \\
timeFirstDayInQuarter & First day in a given quarter \\
timeLastDayInQuarter & Last day in a given quarter \\
timeNdayOnOrAfter & Day "on-or-after" n-days \\
timeNdayOnOrBefore & Day "on-or-before" n-days \\
timeNthNdayInMonth & N-th occurrence of a n-day \\
& in month \\
timeLastNdayInMonth & Last n-day in month \\
\hline
\end{tabular}

\begin{tabular}{|c|c|}
\hline \multicolumn{2}{|l|}{ 2. List of holidays } \\
\hline Advent1st & JPBunkaNoHi \\
\hline Advent2nd & JPChildrensDay \\
\hline Advent3rd & JPComingofAgeDay \\
\hline Advent 4th & JPConstitutionDay \\
\hline Allsaints & JPEmperorsBirthday \\
\hline Allsouls & JPGantan \\
\hline Annunciation & JPGreeneryDay \\
\hline Ascension & JPHealthandSportsDay \\
\hline AshWednesday & JPKeirounOhi \\
\hline AssumptionofMary & JPKenkokuKinenNoHi \\
\hline BirthofVirginMary & JPKenpouKinenBi \\
\hline BoxingDay & JPKinrouKanshaNoHi \\
\hline CACanadaDay & JPKodomoNoHi \\
\hline CACivicProvincialHoliday & JPKokuminNoKyujitu \\
\hline CALabourDay & JPMarineDay \\
\hline CAThanksgivingDay & JPMidoriNoHi \\
\hline CAVictoriaDay & JPNatFoundationDay \\
\hline CHAscension & JPNationHoliday \\
\hline CHBerchtoldsDay & JPNationalCultureDay \\
\hline CHConfederationDay & JPNewYearsDay \\
\hline CHKnabenschiessen & JPRespectForTheAgedDay \\
\hline CHSechselaeuten & JPSeijinNoHi \\
\hline CaRemembranceDay & JPShuubunNoHi \\
\hline CelebrationofHolyCross & JPTaiikuNoHi \\
\hline ChristTheKing & JPTennouTanjyouBi \\
\hline ChristmasDay & JPThanksgivingDay \\
\hline ChristmasEve & JPUmiNoHi \\
\hline CorpusChristi & LaborDay \\
\hline DEAscension & MassofArchangels \\
\hline DEChristmasEve & NewYearsDay \\
\hline DECorpusChristi & PalmSunday \\
\hline DEGermanUnity & Pentecost \\
\hline DENewYearsEve & PentecostMonday \\
\hline Easter & PresentationofLord \\
\hline EasterMonday & Quinquagesima \\
\hline EasterSunday & RogationSunday \\
\hline Epiphany & Septuagesima \\
\hline FRAllSaints & SolemnityofMary \\
\hline FRArmisticeDay & TransfigurationofLord \\
\hline FRAscension & TrinitySunday \\
\hline FRAssumptionVirginMary & USCPulaskisBirthday \\
\hline FRBastilleDay & USChristmasDay \\
\hline FRFetDeLaVictoire1945 & USColumbusDay \\
\hline GBBankHoliday & USDecorationMemorialDay \\
\hline GBMayDay & USElectionDay \\
\hline GBMilleniumDay & USGoodFriday \\
\hline GBSummerBankHoliday & USInaugurationDay \\
\hline GoodFriday & USIndependenceDay \\
\hline ITAllSaints & USLaborDay \\
\hline ITAssumptionOfVirginMary & USLincolnsBirthday \\
\hline ITEpiphany & USMLKingsBirthday \\
\hline ITImmaculateConception & USMemorialDay \\
\hline ITLiberationDay & USNewYearsDay \\
\hline ITStAmrose & USPresidentsDay \\
\hline JPAutumnalEquinox & USThanksgivingDay \\
\hline JPBankHolidayDec31 & USVeteransDay \\
\hline JPBankHolidayJan2 & USWashingtonsBirthday \\
\hline JPBankHolidayJan3 & \\
\hline
\end{tabular}

